Relations industrielles

Industrial Relations

\title{
La qualification des cadres hiérarchiques par le Code du travail
}

\section{Line Management under the Quebec Labor Code}

\section{Rodrigue Blouin}

Volume 30, numéro 3, 1975

URI : https://id.erudit.org/iderudit/028636ar

DOI : https://doi.org/10.7202/028636ar

Aller au sommaire du numéro

Éditeur(s)

Département des relations industrielles de l'Université Laval

ISSN

0034-379X (imprimé)

1703-8138 (numérique)

Découvrir la revue

Citer cet article

Blouin, R. (1975). La qualification des cadres hiérarchiques par le Code du travail. Relations industrielles / Industrial Relations, 30(3), 478-512.

https://doi.org/10.7202/028636ar

\section{Résumé de l'article}

L'auteur analyse la méthode par laquelle les commissaires-enquêteurs et le Tribunal du travail déterminent si un employé peut-être qualifié de cadre hiérarchique au sens de l'article $\mathrm{lm}$ ) $1^{\circ}$ duCode du travail. Après avoir présenté sommairement le critère de fond retenu par les tribunaux et son fondement historique, il passe à l'étude des éléments constitutifs de ce critère: l'exercice de l'autorité hiérarchique ; par délégation de l'employeur ; avec liberté de manoeuvre. L'étude est complétée par une présentation de l'exercice de l'autorité hiérarchique à divers palliers d'action.
Tous droits réservés (C) Département des relations industrielles de l'Université Laval, 1975
Ce document est protégé par la loi sur le droit d'auteur. L'utilisation des services d'Érudit (y compris la reproduction) est assujettie à sa politique d'utilisation que vous pouvez consulter en ligne. 


\section{La qualification des cadres}

\section{hiérarchiques par le Code du travail}

\section{Rodrigue Blouin}

L'auteur analyse la méthode par laquelle les commissaires-enquêteurs et le Tribunal du travail déterminent si un employé peut-être qualifié de cadre hiérarchique au sens de l'article $1 \mathrm{~m}) 1^{\circ} d u$ Code du travail. Après avoir présenté sommairement le critère de fond retenu par les tribunaux et son fondement historique, il passe à l'étude des éléments constitutifs de ce critère: l'exercice de l'autorité hiérarchique; par délégation de l'employeur; avec liberté de manoeuvre. L'étude est complétée par une présentation de l'exercice de l'autorité hiérarchique à divers palliers d'action.

Le Code du travail édicte que le mot salarié «ne comprend pas» 1 «une personne qui, au jugement du commissaire-enquêteur, est employée à titre de gérant, surintendant, contremaître ou représentant de l'employeur dans ses relations avec ses salariés » ${ }^{2}$. Selon les décisions des commissaires-enquêteurs et, en appel, du Tribunal du travail, ces titres en soi et pris isolément ne sont pas importants. La loi vise en effet par ces titres une catégorie spécifique d'employés, catégorie que l'on ne saurait identifier que par la mise en relief d'un critère de fond. La position jurisprudentielle se justifie tout à la fois au plan juridique et au plan pratique. Au plan juridique, la technique utilisée par le législateur est une définition par énumération. Ce procédé «signifie que au lieu d'énoncer un principe, on en donne des exemples ${ }^{3}$. Les titres énumérés à l'article $1 \mathrm{~m}) 1^{\circ}$ ne sont donc que des exemples d'un principe général qui doit présider à l'interprétation du texte. Au plan pratique, ces différents titres sont représentatifs

BLOUIN, R., professeur-adjoint, Département des relations industrielles, Université Laval, Québec.

1 S.R.Q. 1964 , c. 141 , art. $1 \mathrm{~m})$.

2 Ibidem, art. $1 \mathrm{~m}) 1^{\circ}$.

3 L. P. PIGEON, Rédaction et Interprétation des lois, Éditeur officiel du Québec, 1965, Québec, à p. 21. 
d'une réalité mouvante d'une entreprise à une autre, réalité qui ne saurait être traduite que par une norme de principe qui seule peut satisfaire les situations les plus disparates et dont on doit faire application en regard de chaque cas d'espèce.

Les propos qui vont suivre seront consacrés à l'analyse du critère retenu par les tribunaux du travail. Une mise en contexte sera en tout premier lieu consacrée à rappeler l'exigence du critère de fond, sa formulation et son fondement (en I). Ces propos préliminaires attesteront que le critère est axé exclusivement sur l'identification des employés qui sont habilités à exercer l'autorité patronale avec liberté de manoeuvre. L'étude des trois éléments constitutifs de ce critère fera l'objet du coeur de l'exposé. Le premier élément tient à la nature de l'autorité exercée (en II) : seule l'exercice de l'autorité hiérarchique, qui exclut de sa compréhension l'autorité professionnelle ou technique et l'autorité de consultation, permet initialement de procéder à la qualification des membres de la gérance ou cadres hiérarchiques ${ }^{4}$. Le second élément met en cause la source justificative de l'exercice de l'autorité hiérarchique (en III) : l'employé doit exercer au nom et pour le compte de l'employeur, par délégation, l'autorité ; il ne doit pas s'arroger la prérogative patronale. Enfin, le dernier élément a pour objectif essentiel de préciser les conditions d'exercice de l'autorité patronale (en IV) : l'employé doit l'exercer avec une liberté de manoeuvre comportant un degré d'initiative, de jugement et de responsabilité se rapprochant de celui qui caractérise la décision patronale. Nous terminerons l'exposé par des propos complémentaires où il sera question de la répartition de l'autorité hiérarchique à différents palliers d'exercice (en V); dans cette partie de l'étude nous aborderons les notions composantes des termes génériques membres de la gérance ou cadres hiérarchiques.

\section{I - LE CRITÈRE DE FOND}

C'est par la technique de la qualification directe que les tribunaux procèdent à l'identification des employés visés par l'article $1 \mathrm{~m}$ ) $1^{\circ}$. Il s'agit d'un procédé par lequel les commissaires-enquêteurs et les juges recherchent si le travailleur qui se prétend un membre de la gérance mé-

4 Nous utiliserons au cours de l'exposé les expressions membres de la gérance et cadres hiérarchiques comme terme générique ou collectif de l'expression "gérant, surintendant, contre-maître ou représentant de l'employeur dans ses relations avec ses salariés. 
rite ou non cette qualification par l'examen direct de sa situation dans l'entreprise, indépendamment du titre qui coiffe sa fonction (en A). Cette démarche implique l'articulation d'un critère de fond qui trouve son fondement et sa justification dans le contexte historique qui a présidé à l'aménagement des rapports collectifs du travail (en B).

\section{A) Le procédé de qualification}

Les titres mentionnés à l'article $1 \mathrm{~m}) 1^{\circ}$ ne sont pas en soi importants. Ce n'est pas en effet le titre que l'employeur a octroyé à un poste de travail ou à une fonction qui fait de son titulaire un membre de la gérance 5 . Ce sont les fonctions accomplies par l'employé et la nature des responsabilités en relation avec les tâches qui permettent de déterminer s'il y a réellement participation aux activités caractéristiques du management ${ }^{6}$. Si l'on préfère, les fonctions et responsabilités confiées à un employé constituent une question de fait qui doit être appréciée par le tribunal selon le contenu objectif du poste de travail indépendamment du titre qui y est greffé ${ }^{7}$. Deux conséquences découlent dès lors de cette approche.

En premier lieu, la force probante des titres est, aux yeux des tribunaux, très limitée et peut être renversée ${ }^{8}$. La raison est que l'attribution des titres peut être superficielle, voire incertaine. Des exemples tirés de la pratique montrent aisément que des travailleurs n'ayant pas un de ces titres peuvent se comporter en véritables représentants de l'employeur tandis qu'à l'inverse d'autres employés s'identifiant par des titres ronflants ne sont que des exécutants. Dès lors, le fait que l'employeur «a saupoudré des titres sur la tête de ses salariés sans leur donner les respon-

5 Gasoline Stations Ltd. et J. L. PARÉ, (1971) T. T. 321, à p. 322. Hôpital Saint-Rédempteur de Matane et le Syndicat des employés de l'hôpital Saint-Rédempteur de Matane, (1971), T. T. 73, aux pp. 75, 76. Syndicat national des employés de la Crèche d'Youville et Crèche d'Youville, (1971) T. T. 50, aux pp. 52-53. Métallurgistes-Unis d'Amérique, loc. 4278, et Noranda Mines Ltd., (1969), D.C.R.T. 232, à p. 234.

\section{Ibidem.}

7 Ibidem. Alliance des infirmières de Montréal et Hôpital Charles Lemoyne, (1970) C.E. 499, à p. 501. GAGNON, LEBEL et VERGE, Droit du travail en vigueur au Québec, P.U.L., Québec, 1971, à p. 128.

$8 \mathrm{La}$ Cie Atlantic Ltée et Syndicat des employés de bureau Atlantic de Rimouski, (1971) T.T. 335, à p. 337. Gasoline Station Ltd. c. J. L. PARÉ, (1971) T.T. 321, à p. 322 . 
sabilités équivalentes $\gg$ à celles reconnues aux membres de la gérance ne peut être déterminant. Inversément, une fonction qui n'est libellée d'aucun de ces titres peut fort bien en être une de la gérance en raison des attributions qui la caractérisent ${ }^{10}$.

En second lieu, l'entente entre les parties sur la nature d'un poste de travail ne peut avoir pour effet en elle-même d'empêcher les tribunaux d'aller au-delà de l'accord et d'examiner au fond les caractères effectifs de la fonction ${ }^{11}$. Cette position se justifie par le fait «que l'accord des parties n'est pas prépondérant sur la loi ${ }^{12}$. La volonté bilatérale n'est déclaratoire de droit que si elle se fonde sur des critères objectifs ${ }^{13}$ constatés par les tribunaux ${ }^{14}$. Même une corporation municipale ne peut par règlement ou autrement "s'inscrire hors du champ d'application du Code du travail sans un texte spécifique l'autorisant ${ }^{15}$, comme par exemple, désigner par résolution une fonction comme en étant une de direction ${ }^{16}$. Dans la même mesure, un document signé par un employé attestant qu'il est un gérant ne saurait être, en soi, déterminant ${ }^{17}$. Les tribunaux conservent dans ces circonstances leur «compétence pour apprécier au fond la nature de la fonction et les responsabilités qui y sont attachées » ${ }^{18}$. Cette analyse au fond a pour objectif essentiel d'identifier ceux qui, au sein de l'entreprise, personnifient réellement l'employeur. L'articulation du critère de fond qui permet de procéder à cette identification trouve son fondement et sa justification dans le contexte historique.

\section{Ibidem.}

10 L'Association des principaux d'écoles de Québec et la Commission des écoles catholiques de Québec, (1966) R.D.T. 321, à p. 333.

11 Syndicat canadien des officiers de marine marchande et International Union of District 50, United Mines Workers of America, et Place Victoria Saint-Jacques, (1971) T.T. 163, à p. 165. Pasolds (Canada) Ltd. et Ouvriers unis du textile d'Amérique, (1970) T.T. à p. 30.

12 Le Syndicat des employés de l'Hôtel-Dieu de Lévis (C.S.N.) et Hôpital Hôtel-Dieu de Lévis, (1974) T.T. 67, à p. 71.

13 Syndicat canadien des officiers de Marine marchande et la Traverse de Lévis, (1970) T.T. 13, à p. 15.

14 Voir références 11,12 et 13.

15 Ville de Montréal et Syndicat des fonctionnaires municipaux de Montréal, (1974) T.T. 410, à p. 413.

16 Ibidem, aux p. 412, 413. Syndicat canadien de la fonction publique et Ville de Vimont, (1966) R.D.T. 288, à p. 297.

17 Salaison Her Pack Ltée et N. Tétrault, (1971) T.T. 243, à p. 246.

18 Voir note 15 , à p. 412. 


\section{B) Le contexte historique explicatif du critère}

Pour la compréhension du Code du travail, il faut au départ se rappeler que l'arrière-plan qui permet de donner la mesure exacte de l'intention du législateur en matière d'aménagement des rapports collectifs du travail, n'est pas fondamentalement le contexte historique qui prévalait à l'époque de l'adoption du Code, mais celui de la Loi des relations ouvrières ${ }^{19}$. Le Code ne représente en effet que la synthèse de cette dernière Loi et des autres lois adoptées parallèlement ${ }^{20}$. Il est resté sous la coupe de la Loi des relations ouvrières en ce qui regarde l'économie et la philosophie sous-tendant l'ordonnancement juridique des relations collectives du travail ${ }^{21}$. Jusqu'à l'adoption de la Loi des relations ouvrières, les rapports de travail étaient demeurés le plus souvent sur le plan strictement individuel. La transposition fondamentale de ces rapports sur le plan collectif a été conçue par la Loi, puis reprise par le Code, dans et pour une économie à caractère libéral et en fonction notamment d'une préoccupation majeure et fondamentale : réprimer les abus engendrés par le système des rapports individuels de travail en rétablissant l'équilibre des parties à la négociation mais en moulant cet équilibre en fonction de la réalité administrative des entreprises de l'époque ${ }^{22}$. Fondamentale-

19 S.R.Q. 1941, c. 162 A ; bien que reproduite dans les statuts de 1941, cette loi a été édictée par 8 Geo. VI, c. 30 (1944).

20 Loi des différends ouvriers S.R.Q. 1941, c. 167. Loi concernant les enquêtes en matière de différends industriels, S.R.Q., 1941, c. 168. Loi des différends entre les services publics et leurs salariés, S.R.Q., 1941 c. 169. Loi concernant les corporations municipales et scolaires de leurs employés, 13, Geo. VI, c. $26 \ldots$

21 À titre d'illustration, la définition du salarié à l'article $1 \mathrm{~m}$ ) du Code a le même sens et la même portée que celle donnée à l'article 2 a de la Loi : Food Drivers Commission Salesmen, Dairy and Ice Cream Workers, local Union no. 973, and J.J. Joubert, D.C.D.R.T., no. 1638 - 10, à p. 3. D'autre part, le texte $1 \mathrm{~m}) 1^{\circ} \mathrm{du}$ Code est identique dans sa formulation à celui de l'article 2 a souspar. 1 de la Loi et vise en conséquence les mêmes employés : L'Association des principaux d'écoles de Québec et La Commission des écoles catholiques de Québec, (1966) R.D.T. 321, à p. 324.

22 Plusieurs auteurs ont souligné cette mise en situation en fonction de la réalité administrative de l'époque : R. CHARTIER, Le syndicalisme est-il la réponse aux problèmes des cadres? (1971) 26 Rel. Ind. 421 ; Le syndicalisme de cadres et la législation québécoise du travail, (1965) 20 Rel. Ind. 278. J. R. CARDIN, «Une montée inéluctable en économie moderne: le syndicalisme de cadres», Cadres, vol. I, no. 6 , p. 7. Cette réalité pouvait seule semble-t-il, dans l'optique de l'époque, assurer le sain dualisme patronat-salariat recherché par les lois du travail: "Le dualisme patronat-salariat, non pas dans un but de lutte mais en vue de réaliser un équilibre favorisant des relations contractuelles justes, est à la base de ces lois »: L'Association des principaux d'écoles de Québec et la Commission des écoles catholiques de Québec, (1966) R.D.T. 321, à p. 324. 
ment, le schéma des titres énumérés à l'article $1 \mathrm{~m}) 1^{\circ}$ ne fait que reproduire les principes d'organisation des entreprises à l'époque de la production industrielle ${ }^{23}$. Le Code retient et met en évidence l'idée fondamentale issue de la notion d'organisation et selon laquelle les entreprises doivent être structurées à deux niveaux de fonctionnement : niveau de la direction d'une part et, d'autre part, niveau de l'exécution ${ }^{24}$. Par le jeu de l'article $1 \mathrm{~m}$ ) d'une part et, d'autre part, des articles $1 \mathrm{~m}$ ) $1^{\circ}$ et $1 \mathrm{~m}) 2^{\circ}$, le législateur affirmait que sur le plan juridique, comme d'ailleurs sur le plan des techniques d'organisation, l'entreprise doit être perçue comme structurée à deux niveaux. Le législateur a voulu ainsi attacher des conséquences juridiques particulières au fait que l'autorité patronale est concentrée entre les mains des dirigeants de l'organisation scientifique des entreprises et réparties entre eux de façon hiérarchique à divers niveaux ou palliers d'exercice ${ }^{25}$. L'article $1 \mathrm{~m}$ ) $1^{\circ}$ vise en somme les employés qui personnifient dans l'entreprise l'autorité du patron ${ }^{26}$.

On ne doit pas pour autant en conclure que la notion des membres de la gérance au Code du travail vise tous ceux qui sont considérés comme participants de la direction en science administrative et organisationnelle de l'entreprise. Ces dernières sciences ont en effet une vision globaliste de la notion de direction et qui inclut tous ceux «qui com-

23 Parmi les principaux artisans des théories d'organisation des entreprises à l'époque, on doit souligner F. Taylor et $\mathrm{H}$. Fayol ainsi que les auteurs qui ont contribué à l'école classique d'organisation ou de gestion scientifique des entreprises. F. TAYLOR, La Direction scientifique des entreprises, Dunod, Paris, 1957 ; Principes d'organisation scientifique, Dunod, Paris, 1927 ;La Direction des ateliers, Dunod, Paris, 1913. H. FAYOL, Administration industrielle et générale, Dunod, Paris, 1956. Pour des exposés sur l'école scientifique : M. GRENIER et B. MONTEIL, Principes de management, P.U.Q., Québec, 1971, pp. 316 à 328. J. TESIOROWSKI, Organisation et gestion de l'entreprise, Beauchemin, Montréal, pp. 237 et ss. BELANGER et KHOUNY, L'Administration-Principes et Fonctions, McGraw-Hill, Montréal, 1970, pp. 163 et ss. L. PICARD, L'évolution des théories en administration, Actualité économique, 1965, oct.-déc., pp. 383 à 417.

24 Voir les auteurs cités à la note 22 .

25 Sur la monopolisation et la répartition de l'autorité patronale, consultez les auteurs cités à la note 23 .

26 R. GAGNON, L. LEBEL et P. VERGE, Droit du travail en vigueur au Québec, op. cit., note 7, à p. 128. R. LEMOINE, Le gérant, le surintendant, le contremaître et le représentant de l'employeur dans ses relations avec ses salariés, (1968) R.D.T. 449, à p. 445. L. M. BEAULIEU, Les conflits de droit dans les rapports collectifs du travail, P.U.L., Québec, 1955, aux pp. 181 et 182. 
mandent à quelque niveau que ce soit ${ }^{27}$. Elles ont une approche qui considère la direction comme un «bloc monolithique s'étendant de la haute direction (...) au détenteur de la moindre parcelle d'autorité de l'entreprise : (...) chef de groupe, etc $\gg 28$. Pour sa part, le législateur n'a voulu viser que ceux qui participent réellement à la direction. Le critère de fond dégagé par les tribunaux, l'exercice autorisé de l'autorité patronale avec liberté de manoeuvre, est en somme destiné à rendre compte que le législateur a entendu se préoccuper uniquement de la philosophie relative à la délégation de l'autorité patronale indépendamment des structures pensées à l'époque de la production industrielle et peu importe l'évolution dans le temps de l'aménagement de ces structures. Ce qui importe est essentiellement la nature des fonctions et responsabilités, lesquelles doivent être significatives de la notion d'autorité patronale. Il convient maintenant de voir à quelles exigences la notion d'autorité patronale donnent lieu. Ces exigences sont relatives à la nature même de l'autorité, à la source justificative et aux conditions d'exercice de l'autorité.

\section{Ili- LA NATURE DE L'AUTORITÉ}

On a de l'autorité diverses conceptions. Ainsi, on parle par exemple occasionnellement de «l'autorité personnelle », c'est-à-dire une forme de confiance ou de regret qu'impose un individu en raison de sa compétence. Cette forme d'autorité n'intéresse pas le Code du travail. Parce que le substratum historique ne permet de comprendre par l'article $1 \mathrm{~m}$ ) $1^{\circ}$ que les dirigeants de l'organisation scientifique des entreprises, les tribunaux ont dégagé qu'un dirigeant ne saurait exister que dans la mesure où un employé exerce la fonction essentielle et spécifique de l'employeur. Cette fonction est celle qui consiste à prendre des décisions contraignantes. Elle est qualifiée de «pouvoir de commandement», plus exactement d'«autorité hiérarchique ou patronale». Cette autorité a ses exigences propres de qualification (en A) et exclut de sa compréhension de nombreuses formes d'autorité (en B).

27 Certains auteurs semblent affirmer que la législation aurait le même effet : voir les auteurs cités à la note 22 . Nous démontrerons en cours d'exposé que rien n'est moins exact.

28 J. R. CARDIN, «Une montée inéluctable en économie moderne : le syndicalisme de cadres », op. cit., note 22 , à p. 7 . Ces propos appellent le même commentaire que celui fait à la note précédente. 


\section{A) La qualification de l'autorité hiérarchique}

La qualification de l'autorité patronale exige de la situer en relation avec l'organisation administrative. Le droit a en effet articulé la notion d'autorité hiérarchique en prenant pour assiette les fonctions patronales élaborées par la science administrative. Toute entreprise doit orienter ses diverses activités vers un but commun, vers une finalité économique ou sociale. Elle doit à cet effet gérer. Selon la présentation classique, gérer c'est tout à la fois organiser, coordonner et planifier, informer, contrôler et commander ${ }^{29}$. On parlera de gestion financière, de gestion du personnel, de gestion commerciale, etc. ${ }^{30}$. Pour le droit, seule la Gestion, en soi et peu importe la zone d'activité où elle s'exerce (finance, personnel, etc.), importe dans le processus de qualification des membres de la gérance. Pour les fins de cette qualification, l'approche juridique regroupe les différentes activités composantes de la Gestion en deux catégories : d'une part les fonctions de moyen (organisation, coordination et planification, information, contrôle) et, d'autre part, une fonction de résultat (commandement).

Sous l'angle juridique, la fonction de résultat, la seule qui nous intéresse ici, apparaît comme l'élément caractéristique et spécifique de la qualification des membres de la gérance. Cette fonction est le «commandement». Le commandement est l'acte décisionnel par lequel les niveaux hiérarchiques supérieurs dans l'entreprise déclenchent et orientent les activités des niveaux inférieurs et, à la limite, des exécutants vers un but commun. C'est le fait de donner des ordres et d'être obéi, le fait de prendre des décisions qui obligent et contraignent. L'autorité légitime cette action ${ }^{31}$. Elle permet de donner des ordres, de décider ; elle autorise à sanctionner en cas de désobéissance, à rectifier en cas de non-conformité aux décisions. Dans la pratique quotidienne, le commandement se manifeste à divers palliers ou niveaux d'action. Selon le niveau, le commandement emporte un acte final qui déclenche l'activité du niveau inférieur. Le commandement est en conséquence hiérarchisé. L'autorité

29 M. GRENIER et B. MONTEIL, Principes de management, op. cit., note 23, à 93.

30 Pour des exemples d'aménagements variés des zones de gestion, on pourra confronter : BÉLANGER et KHOUNY, L'Administration-Principes et Fonctions, op. cit., note 23, à p. 163. GRENIER et MONTEIL, Principes de management, op cit., note 23, à p. 378 .

31 L. SALLERON, Autorité et commandement dans l'entreprise, Éditions de l'entreprise moderne, Paris, 1960, p. 35. 
qui s'échelonne corrélativement tout au long de la chaîne de commandement est hiérarchisée. Il n'est donc pas nécessaire qu'un employé exerce toutes les fonctions susceptibles d'être accomplies par un employeur en matière de Gestion pour être considéré comme un dirigeant dans l'entreprise ${ }^{32}$. L'exercice de tous les pouvoirs et fonctions amènerait la «présence d'un gérant-général dont les attributs n'auraient de limite que celles de l'employeur lui-même» ${ }^{33}$. Il suffit mais il est impérieux d'exercer le commandement ou partie réelle du pouvoir de commandement en matière d'organisation, de coordination et de planification, d'information et de contrôle, dans un secteur d'activités (finances, ressources humaines, etc.) indépendamment du lieu d'exercice (département, service, etc.). Il faut donc être titulaire de l'autorité patronale, être autorisé à prendre des décisions qui obligent les subalternes ou l'entreprise dans ses activités ${ }^{34}$. Ainsi la participation à la conception des décisions administratives, à la planification ou à la définition des politiques peut constituer un motif d'inclusion à la notion des membres de la gérance, mais dans la mesure où ces actions sont sous-tendues par le pouvoir potentiel de l'exercice du commandement : il faut que l'employé participe et collabore réellement à la prise de décision qui oblige et qui se présente comme les voies d'action à suivre dans l'accomplissement des activités de l'entreprise. L'exercice d'activités de planification, d'organisation et autres de même nature, sous forme de tâches professionnelles, techniques ou consultatives d'où la participation à la prise de décision en soi est exclue, entraîne la présence de formes d'autorité qui ne composent pas avec la notion d'autorité hiérarchique ou patronale.

En somme, l'autorité hiérarchique se confond avec le «droit de prendre des décisions », «droit de diriger, contrôler et surveiller ». $\mathrm{Ce}$ droit seul permet d'avoir une influence certaine dans le processus conduisant à la formulation et à l'élaboration des buts recherchés par l'en-

32 Résidence Dorchester et Union des employés de service, loc. 298, (1973) T.T. 77, à p. 79. Association des employés de la Société Saint-Vincent-de-Paul et Société Saint-Vincent-de-Paul (1970) T.T. 431, à p. 434. P. Fisher et la Centrale du livre inc., (1969) D.C.R.T. 213, à p. 215. La Ville de Sainte-Foy et le Syndicat des fonctionnaires municipaux de la Ville de Sainte-Foy, (1967) R.D.T. 205, J. B. Laliberté Ltée et Association des commis de détail, (1967) R.D.T. 246, aux p. 248 et 249. R. GAGNON, L. LEBEL et P. VERGE, Droit du travail en vigueur au Québec, op. cit., note 7, aux pp. 127 et 128.

33 Ibidem.

34 Buy-Well Food Market Inc. et M. Godin, (1971) T.T. 302, à p. 303. Syndicat professionnel des diététistes du Québec et Hôpital Saint-Jean de Dieu, (1971) T.T. 241, à p. 242. Voir de même la jurisprudence citée à la note 32 . 
treprise et des moyens d'action pour atteindre ces buts. Ce droit ne peut exister que dans la mesure où son véritable titulaire, l'employeur, le délègue. C'est pourquoi conviendra-t-il d'élaborer sur la délégation. Auparavant, il importe de cerner certaines formes d'autorité confondantes qui ne relèvent pas de la compréhension de l'autorité patronale.

\section{B) Les formes d'autorité exclues}

La nécessité pour les entreprises de faire appel à un personnel de plus en plus spécialisé dans diverses disciplines scientifiques, techniques ou professionnelles, a généralement entraîné la mise en place d'une structure professionnelle où certains employés disposent à l'égard de d'autres d'une forme d'autorité qualifiée d'autorité professionnelle ou technique. Pour les mêmes raisons, l'entreprise fait souvent appel dans l'élaboration de ses politiques à des spécialistes employés à titre de cadres-conseils, spécialistes titulaires de ce qu'il est convenu d'appeler l'autorité de réflexion. Ces formes d'autorité ne relèvent pas en soi et à leur état pur de la notion d'autorité hiérarchique.

\section{L'AUTORITÉ PROFESSIONNELLE ET TECHNIQUE}

Les tribunaux ont à maintes reprises rappelé que les rapports d'ordre purement professionnel ou technique, bien qu'ils comportent nécessairement une incidence de direction et impliquent une certaine forme d'autorité, n'emportent pas par eux-mêmes l'exercice de l'autorité hiérarchique ${ }^{35}$. Trois raisons fondamentales expliquent cette approche: le rapport de direction professionnelle ou technique tient uniquement à l'expérience ou à la compétence plus prononcée du titulaire de l'autorité sur ses subordonnés; ce rapport porte sur des matières qui ne sont pas d'ordre administratif; et, enfin, il s'inscrit dans le cadre de la pure exécution de politiques élaborées selon un processus décisionnel d'où est exclu le détenteur du pouvoir professionnel ou technique ${ }^{36}$. L'autorité

35 Maison Ste-Clothilde Inc. et le Syndicat canadien de la fonction publique, loc. 1554, (1973) T.T. 446. Institut Philippe Pinel de Montréal et Syndicat national des employés de l'Institut Philippe Pinel (1972) T.T. 67. Cité d'Alma et Syndicat national des employés municipaux d'Alma, (1972) T.T. 183. Service familial de la rive Sud et Syndicat des employés du Service familial de la rive Sud, (1972) T.T. 71. On pourra aussi consulter : (1971) T.T. 315; (1971) T.T. 243 ; (1971) T.T. 241 ; (1971) T.T. 216 ; (1971) T.T. 163 ; (1971) T.T. 14 ; (1970) T.T. 413.

36 Ibidem, Hôpital St-Eusèbe de Joliette et Syndicat catholique national des employés des institutions religieuses du diocèse de Joliette, (1970) T.T. 413, Union Internationale des employés professionnels et de bureau, loc. 57, et Commission scolaire régionale de Chambly, (1971) C.E. 977. Alliance des infirmières de Montréal et Hôpital Charles Lemoyne, (1970) C.E. 499. On pourra de même consulter : (1971) C.E. 765 ; (1970) C.E. 434. 
professionnelle ou technique est en somme une forme d'autorité qui se manifeste dans un cadre autre que celui d'une participation à la formulation et à la mise en application des buts et voies d'action de l'entreprise. En d'autres termes, elle ne participe pas de la décision administrative ${ }^{37}$.

L'exercice à l'état pur de l'autorité professionnelle et technique ne permet donc pas de procéder à la qualification d'un employé comme membre de la gérance. Il peut en être autrement lorsque cet exercice s'accompagne du pouvoir décisionnel (autorité patronale). La prédominance de l'autorité hiérarchique dans l'exercice d'une fonction qui comporte aussi des tâches de direction professionnelle ou technique fait du titulaire de cette fonction un véritable membre de la gérance ${ }^{38}$, tandis que la solution inverse s'impose au cas contraire ${ }^{39}$. La démarche s'explique par le fait que c'est l'ensemble des caractéristiques de la fonction et du travail qui permet de déceler le véritable statut de l'employé 40 .

\section{L'AUTORITÉ DE RÉFLEXION (CADRE-CONSEIL)}

La solution à être préconisée en matière de qualification des titulaires de l'autorité de réflexion ne peut prendre appui sur la jurisprudence vu l'absence de débat de fond sur la matière. Au plan académique, les

37 L'autorité professionnelle et technique doit être comprise en termes de rapport d'ordre professionnel et ne pas être confondue avec le fait des connaissances techniques ou professionnelles en tant que telles et nécessaires à l'exercice de l'autorité hiérarchique. Consultez: Syndicat des diététistes du Québec et Hôpital Maisonneuve, (1972) T.T. 366. Unité locale de scieries de la fraternité des charpentiers-menuisiers d'Amérique et la Compagnie Eagle Lumber Inc., (1971) C.E. 451.

38 Syndicat des fonctionnaires municipaux de Ville d'Anjou et Ville d'Anjou, (1972) T.T. 393. R.C.A. Engineers and Scientists Association et R.C.A. Ltd., (1972) T.T. 191. Syndicat des employés de bureau de la mine Campbell et Campbell Chibougamau Mine Ltd., (1972) T.T. 53.

39 Cité d'Alma et Syndicat national des employés municipaux d'Alma, (1972) T.T. 183. Syndicat professionnel des diététistes du Québec et Hôpital St-Sacrement, (1972) T.T. 85. Service social de l'Outaouais et le Syndicat des professeurs du Service social de l'Outaouais, (1972) T.T. 63.

40 Syndicat des employés de l'Hôtel-Dieu de Lévis et Hôtel-Dieu de Lévis, (1974) T.T. 67, à p. 70. Syndicat des fonctionnaires municipaux de Ville d'Anjou et Ville d'Anjou, (1972) T.T. 393, à 396. On pourra consulter sur le problème du cumul d'une fonction de salarié (autorité professionnelle) et d'une fonction de membre de la gérance la jurisprudence citée aux notes 57 à 62 . On pourra de même consulter la jurisprudence citée à la note 56 pour l'exercice occasionnel de la fonction de gérance. 
cadres-conseils peuvent être sommairement présentés comme ces employés qui ne détiennent pas une fonction de direction ou d'administration (selon la ligne d'autorité «line») mais qui assument une responsabilité scientifique (fonction «staff ») ${ }^{41}$. Les cadres-conseils sont donc les employés qui ne font que conseiller et orienter, par le poids de leur autorité de réflexion, l'entreprise. Puisque leurs fonctions consistent essentiellement dans la présentation d'analyses, de commentaires, de suggestions, de recommandations et d'alternatives d'action au niveau de la conception, de l'orientation, de la planification, de la recherche, etc., alternatives qui feront l'objet d'un choix par les véritables détenteurs du pouvoir décisionnel, on doit conclure que les tribunaux se prononceront en faveur de l'exclusion de ces cadres du sens et de la portée de l'article $1 \mathrm{~m}) 1^{\circ}$. Cette conclusion s'impose à tout le moins à la lumière des décisions à l'effet que l'autorité professionnelle qui fait de son titulaire un conseiller de la direction n'est pas suffisante pour procéder à la qualification de membre de la gérance ${ }^{42}$, ou encore à l'effet que la simple participation à l'élaboration d'un programme correspondant à une orientation à suivre ne relève pas de la notion de la direction ${ }^{43}$.

On concevra cependant facilement que sur le plan pratique l'exercice de la fonction-conseil existe peu à l'état pur. Elle s'accompagne généralement d'une tranche de l'autorité hiérarchique. L'importance de cette fonction dans le processus de décision, de surveillance et de contrôle des politiques de l'entreprise ne doit pas en effet être minimisée ${ }^{44}$. Si le cadre dit conseil participe réellement aux prises de décision, directement ou indirectement, son véritable statut au sein de l'entreprise relève de celui de membre de la gérance ou cadre hiérarchique (du moins au sens du Code du travail). Dans la même mesure, l'exercice d'une fonction-conseil par rapport aux autorités supérieures mais s'accompagnant d'une autorité dite «line » sur une structure d'exécution ne pourrait que permettre d'en arriver à la conclusion d'un employé visé par l'article $1 \mathrm{~m}$ ) $1^{\circ}$.

41 J. G. RODRIGUE, Les syndicats de cadres au Québec, Les relations $d u$ travail au Canada, U.Q.A.M., fév. 1973, p. 6. Voir de même les auteurs cités à la note 22 .

42 À titre d'illustration: Syndicat professionnel des diététistes du Québec et Hôpital Saint-Jean de Dieu, (1971) T.T. 241, à p. 242.

43 À titre d'illustration : Université du Québec à Montréal et Syndicat des professeurs de l'Université du Québec (Montréal), (1971) T.T. 216, à p. 218.

44 La fonction du Conseil dans le processus de décision et de contrôle, XVIIe Congrès des relations industrielles de l'Université Laval, P.U.L., Québec, 1962. 
Exclues de la compréhension de la notion d'autorité hiérarchique en raison du fait qu'elles ne s'insèrent pas dans le processus de la formulation et de la surveillance des objectifs de l'entreprise et moyens d'action pour les atteindre, l'autorité professionnelle ou technique et l'autorité de réflexion constituent néanmoins des notions d'importance dans la compréhension de l'aménagement des rapports collectifs du travail. Ces notions permettent notamment de faire ressortir que les tribunaux s'en tiennent, à juste titre, aux délimitations imposées par l'organisation scientifique des entreprises pour la compréhension des employés visés à l'article $1 \mathrm{~m}$ ) $1^{\circ}$, employés dits du groupe de la direction. En effet, même si une hiérarchie professionnelle a pris place dans les entreprises par suite de l'apparition de différents phénomènes de travail, comme par exemple l'abandon de la division du travail en tâches parcellaires au profit de nouvelles techniques de répartition des tâches ou encore le déclin de l'exécution purement matérielle au profit d'instruments techniques de travail, les tribunaux ne privent pas pour autant du statut de salarié des employés qui, dans l'optique classique de la gestion scientifique, ne sont pas titulaires de l'autorité déléguée par le patron. Il importe maintenant de préciser quelle importance a la dimension délégation par l'employeur dans la compréhension de la notion d'autorité patronale.

III - LA SOURCE JUSTIFICATIVE DE L'EXERCICE DE L'AUTORITÉ PATRONALE

En reconnaissant à un employé son inclusion au «groupe de la direction » pour les fins de l'aménagement des rapports collectifs du travail, les tribunaux s'autorisent de l'idée selon laquelle cet employé, loin d'être un pur exécutant, accomplit une fonction qui appartient normalement à l'employeur. Trois conséquences en découlent. En premier lieu, il est nécessaire que l'employé soit autorisé à agir au nom et pour le compte de l'employeur; la problématique de l'autorisation met en cause la notion de délégation (en $\mathrm{A}$ ). Deuxièmement, il suffit que l'employé exerce l'autorité déléguée de façon principale ; il n'est pas nécessaire que la fonction soit absorbante (cumul de fonctions) (en B). Enfin, il faut que la fonction soit exercée à travers des structures qui apparaissent comme le véhicule des directives patronales comprises selon le schéma-clé de notre droit du travail, à savoir le dualisme patronat-salariat (direction-exécution) (en C). 


\section{A) La délégation}

Un employé exerce l'autorité hiérarchique quand il agit au nom et pour le compte de l'employeur ${ }^{45}$. Cette exigence implique l'autorisation préalable d'agir ${ }^{46}$, autorisation rendue effective par la délégation. La délégation est « (...) l'acte par lequel le chef transfère une partie de ses attributions à ses subordonnés ${ }^{47}$. Selon les tribunaux, la délégation de l'autorité patronale aux membres de la gérance s'inscrit dans la perception d'une chaîne d'autorité bien définie reliant l'employeur, par les membres de la gérance les uns aux autres, aux exécutants. L'autorité ainsi déléguée n'est que l'autorité patronale initiale. Cette perception a comme corrollaire le concept de l'unité de commandement qui veut que l'autorité mise à la disposition d'un cadre hiérarchique n'est qu'un moyen de simplification des liaisons entre l'employeur et les exécutants. L'autorité qui semble fractionnée dans les faits est au plan juridique monolithique. Cette transmission n'est qu'une technique d'organisation, technique qui n'altère pas la nautre et l'homogénéité de l'autorité patronale. En somme, l'autorité hiérarchique ne saurait exister pour un employé que dans la mesure où il est titulaire d'une délégation. Il n'est cependant pas nécessaire que les délégués obtiennent une approbation expresse pour agir. Pour les tribunaux, l'autorisation d'agir au nom et pour le compte de l'employeur résulte des attributions mêmes des individus. Comme ces attributions sont généralement fonction de deux modes de délégation de l'autorité, soit d'une part le système opérationnel et, d'autre part, le système représentatif, les tribunaux ont formulé certains indices juridiques qui permettent de déterminer si ces systèmes sont réellement opératoires en termes de transmission de l'autorité hiérarchique.

45 R.C.A. Limited et R.C.A. Engineers and Scientists Association. (1971) T.T. 14, aux pp. 22 et 23 -Syndicat des ouvriers de la Régie des Alcools du Québec et la Régie des Alcools du Québec, (1970) C.E. 365, à p. 367 - Le Syndicat national des commis et comptables de St-Joseph d'Alma Inc. et la Coopérative agricole des producteurs d'Alma, (1970) C.E. 970 - Union internationale des travailleurs en boulangerie et en confiserie d'Amérique, loc. 55, et Christie Bread, (Division of Nabisco), (1969) D.C.R.T. 40, à p. 44 - Association des employés des centres en formation professionnelle de la province de Québec et la Commission de formation professionnelle de la main-d'œuvre de la région de Trois-Rivières, (1971), C.E. 705, aux pp. 708 et 709.

\section{Ibidem.}

47 J. TESIOROWSKI, Organisation et gestion, op. cit., note 23, à p. 252. 
Selon ce système, le délégué accomplit ses fonctions conformément à la structure de l'entreprise. L'organigramme prévoit le poste de travail ou encore l'organisation laisse présumer son existence. Le délégué est alors implicitement autorisé à agir : il est investi de l'autorité vis-à-vis ses subordonnés et de la responsabilité envers ses supérieurs. Il n'est pas nécessaire que sa position le situe comme titulaire de l'autorité patronale en regard de l'ensemble de l'entreprise. Il suffit de gérer une division, un département, un service, un groupe de travail indépendant au point de vue opération ${ }^{48}$. La fonction du délégué peut concerner tout aussi bien les rapports externes de l'entreprise (clients, public, etc.) ${ }^{49}$ que les rapports internes ${ }^{50}$. Il n'importe pas dès lors que le délégué soit intégré dans l'élément opérationnel assumant la gestion du personnel. Il suffira de constater que le responsable d'un secteur d'activité, par exemple celui de la gestion financière, possède ou participe, en l'isolant du reste de l'entreprise, à l'autorité décisionnelle en matière de gestion des finances. $\mathrm{Ce}$ responsable représente par rapport aux subalternes l'autorité patronale 51 . Il n'est pas essentiel que cet employé ait du personnel à son service 52 . De même, il n'est pas important, au cas de direction de personnel, que ce dernier soit syndiqué ou ne le soit pas ${ }^{53}$, qu'il soit nombreux ou non ${ }^{54}$. En somme, la délégation opérationnelle fait ressortir que la

48 Association nationale des employés de l'alimentation au détail du Québec Inc. et J. B. Cloutier Inc., (1969) D.C.R.T. 9, à p. 11 - Syndicat des employés du bureau de journaux et la Presse Ltée, (1970) T.T. 246. Ronald Renaud et la Fédération des journalistes canadiens Inc., (1970) C.E. 402 - Syndicat des gérants de districts du journal Le Devoir et Imprimerie Populaire, (1971) C.E. 36 Montreal Newspaper Guild Loc. 111, et Montreal Star Co. Ltd. (1964) R.D.T. 532.

49 Le Syndicat canadien de la fonction publique et la Ville de Vimont, (1966) R.D.T. 282, à p. 299.

50 L'Association des avocats du contentieux de la Ville de Montréal et Ville de Montréal, (1970) C.E. 666 (modifié pour partie sur d'autres aspects : (1970) T.T. 257) - Association des principaux d'écoles de Québec et Commission des écoles catholiques de Québec, (1966) R.D.T. 321.

51 Voir notes 48,49 et 50.

52 The Montreal Newspaper Guild, loc. 111, and Montreal Star Co. Ltd., (1964) R.D.T. 532, aux pp. 540 et 541 - Runkle, J.P., «The Buyer as a Depend Department Manager», dans The Buyer's Manual, National Retail Day Good Association, New York, 1949, à p. 36.

53 J. B. Laliberté Limitée et Association des commis de détail, (1967) R.D.T. 246, aux pp. 246 à 249.

54 Syndicat des fonctionnaires municipaux de Ville d'Anjou et Ville d'Anjou, (1972) T.T. 393 (confirmant 1972 C.E. 244). 
fonction principale de la Gestion (commandement ou pouvoir décisionenl) peut s'exercer en regard de toutes les fonctions de résultat de la Gestion (coordination, planification, etc.) et non seulement en regard de la seule administration de ressources humaines. C'est pourquoi certains auteurs ont-ils pu mentionner, à juste titre, que le législateur «(...) n'a pas établi que le fait de diriger un personnel soit un attribut essentiel de toutes tâches de direction $\gg 55$.

\section{LA DÉLÉGATION OCCASIONNELLE OU SYSTĖME REPRÉSENTATIF DE DÉLEGATION}

Ce système consiste en une délégation occasionnelle confiée à un employé dans des circonstances particulières. Il est ordinairement utilisé pour la transmission d'instructions urgentes et pour la prise de décisions non coutumières. Pour les fins d'application du Code du travail, cette forme d'autorité n'est pas prise en considération. Pour qu'un employé puisse être considéré comme le titulaire d'un poste de gérance, il est nécessaire et essentiel qu'il en exerce les attributions d'une façon continuelle et non seulement occasionnelle ${ }^{56}$. La délégation occasionnelle d'autorité ne doit cependant pas être confondue avec le problème du cumul des fonctions de membres de la gérance et des fonctions de salarié (exécu$\tan$ ).

\section{B) Le cumul des fonctions de la gérance et du salarié}

Quand la fonction d'un membre de la gérance n'est pas absorbante, celui-ci pourra éventuellement exercer les tâches des exécutants. Puisque la loi ne défend pas le cumul des fonctions de gérance et du salarié, ce cumul est en principe juridiquement possible. Les tribunaux québécois ont donné effet à ce principe. C'est l'ensemble des caractéristiques d'un poste de travail qui permet de conclure si son titulaire occupe véritablement ou non un poste de gérance ${ }^{57}$. Dans leur appréciation, les tribu-

55 VERGE et autres, Droit du travail en vigueur au Québec, op. cit., note 7 à p. 127.

56 Hôtel-Dieu d'Arthabaska c. le Syndicat des employés d'Hôpitaux et Hospices du Comté d'Arthabaska, (1970) T.T. 31 à 33. - Ville de Hauterive c. Syndicat national des employés de bureau de la ville de Hauterive, (1971) C.E. 765 - Cité de Hull c. Association des employés de la Cité de Hull Inc., (1971) C.E. 161, à p. 163 - La Ville de Gagnon c. La Fraternité des policiers et pompiers de Ville de Gagnon, (1970) C.E. 107, à p. 109.

57 Lebern Jewellery et Union des employés de commerce, loc. 500, (1973) T.T. 326, à p. 237. Syndicat des fonctionnaires municipaux de Ville d'Anjou et Ville d'Anjou, (1972) T.T. 244. 
naux s'en tiennent à l'étude et à l'analyse des fonctions effectivernent accomplies et non celles susceptibles d'être accomplies à l'avenir ${ }^{58}$. Dès qu'un employé se comporte comme un représentant de la gérance chaque fois que le besoin se fait sentir, la situation est suffisante pour l'exclure de la compréhension du terme salarié ${ }^{59}$. Le fait que les tâches administratives n'absorbent qu'une faible partie du temps de l'employé n'affecte en rien l'autorité qu'il incarne vis-à-vis les salariés ${ }^{60}$. L'exercice occasionnel des fonctions de salarié ne lui fait pas perdre son statut de représentant de l'employeur ${ }^{61}$ lors même que ses fonctions de salarié sont effectuées conformément aux dispositions d'une convention collective ${ }^{62}$.

En somme, lorsque le pouvoir de commandement est exercé en conformité d'une délégation d'autorité patronale, un employé est susceptible d'être qualifié de participant à la gérance. Encore faut-il que la délégation soit en application de structures qui attestent du dualisme directionexécution au sein de l'entreprise.

\section{C) Le dualisme direction-exécution}

Le concept d'autorité hiérarchique est une notion qui donne effet au postulat de l'organisation scientifique voulant que l'entreprise ne puisse opérer et être rentable que si l'on identifie bien les employés-dirigeants (ceux qui commandent) et les salariés-exécutants (ceux qui obéissent). Les structures administratives traditionnelles donnent effet à ce postulat. Dans certaines structures modernes, il arrive que le commandement soit remis entre les mains d'un groupe d'employés tout à la fois dirigeants et

58 Ville de Repentigny et Syndicat des employés de Ville de Repentigny, (1972) T.T. 258.

59 Hôpital Joyce Mémorial c. Syndicat national des employés de l'Hôpital Joyce Mémorial, (1970) T.T. 343.

60 Hôpital Cloutier v. Association professionnelle des physiothérapeutes du Québec et Union des employés de service d'édifices, loc. 298, (1970) T.T. 251 Syndicat des fonctionnaires municipaux de Ville d'Anjou, (1972) T.T. 393.

61 Le Syndicat national des employés de la Société coopérative agricole de Thetford-Mines et Société coopérative agricole de Thetford-Mines, (1971) C.E. 461 à 463 - Union internationale du district 50, travailleurs alliés et techniques des États-Unis et du Canada, local 15469 et D.E.F. Auto Electric Ltd., (1972) C.E. 77 - United Steel Workers of America - Métallurgistes Unis d'Amérique et la Frontière Inc., (1972) C.E. $329-$ Kérillon Canada Ltée et Ouvriers unis du caoutchouc, liège, linoléum et plastique d'Amérique, (1972) T.T. 376.

62 Hôpital du Saint-Sacrement et Alliance professionnelle des para-médicaux de Québec, (1974) T.T. 201, à p. 205. 
exécutants. On parle alors d'auto-commandement collectif, de collégialité, de décision collégiale. Après avoir cerné sommairement cette notion, nous verrons son effet en regard de la qualification des membres de la gérance.

\section{LA NOTION DE DÉCISION COLLÉGIALE}

L'idée générale qui se dégage de la technique d'aménagement des rapports de travail mettant en cause la notion de collégialité réfère à une certaine conception du «groupe social» et postule que l'autorité dans l'entreprise n'est pas autre chose que «(...) l'ensemble des fonctions concourant à l'efficacité du groupe » ${ }^{63}$. L'autorité serait une "fonction collective » remplie de façon diverse par tous les membres du groupe. Appliquée à la science administrative, cette perception a donné lieu à deux postulats. Le premier veut qu'il puisse y avoir des équipes de travail qui fonctionnent sans chef formel désigné. La science dite socio-technique permettrait aux membres des équipes de s'attribuer des tâches, de s'autocontrôler et de s'auto-surveiller, le tout conformément à un objectif final défini par l'employeur ou, dans le cadre de certaines entreprises, par le groupe lui-même. Le deuxième postulat veut que lorsqu'il y a un chef de groupe, ce dernier ne monopolise pas l'autorité mais est uniquement un expert aidant l'équipe à fonctionner pour le compte du groupe tout entier et de l'entreprise. Critiquée par certains auteurs pour le motif qu'il est nécessaire d'avoir une autorité reconnue si l'on veut que les objectifs recherchés soient réalisés ${ }^{64}$, les tribunaux ont néanmoins donné effet à la nouvelle conception là où elle fait l'objet d'expériences administratives.

L'AUTORITE COLLÉGIALE ET LA QUALIFICATION DES MEMBRES DE LA GÉRANCE

Dans un contexte où l'aménagement des structures n'apparaît pas comme le véhicule de la transmission des directives patronales mais se présente plutôt comme une technique destinée à donner effet à la volonté ou décision d'un "groupe social », la participation d'un salarié à la prise de décision collégiale destinée à traduire la volonté du groupe ne lui fait pas perdre son statut de salarié. C'est du moins la solution adoptée par les tribunaux. Nous soulignons ici deux exemples particuliers : les directeurs de département et les vice-doyens qui, à l'Université du Québec, ont été qualifiés de salariés par les tribunaux.

63 J. SALLERON, Autorité et commandement dans l'entreprise, Édition de de l'entreprise moderne, Paris, 1960, aux pp. 62 à 82 (la citation est tirée de la page 64).

64 Ibidem. 
À l'Université du Québec, lorsqu'il s'agit de prendre des décisions qui concernent la vie, les politiques et le fonctionnement de certains départements, l'autorité finale appartient à des assemblées départementales constituées de l'ensemble des membres du corps professoral du département ${ }^{65}$. Ces assemblées élisent un comité exécutif composé d'un directeur et de quatre membres. Le comité a pour fonctions d'élaborer la politique de département et d'en contrôler l'exécution une fois adoptée par l'assemblée. La structure départementale laisse voir que le rôle du directeur, que l'on pourrait concevoir comme participant d'une tâche de la direction dans les structures ordinaires, s'exercent «en exécution» des décisions du département plutôt qu'en fonction des décisions prises par les autorités supérieures de l'Université. Dans un tel contexte, le directeur de département n'est pas en relation d'autorité avec les salariés. Il n'est qu'un agent de coordination et d'encadrement sur le plan professionnel. Il ne peut dès lors être qualifié de cadre hiérarchique, son rôle ne se situant pas dans la ligne d'autorité administrative ${ }^{66}$. De simples pouvoirs de coordination ne sont pas suffisants pour faire acquérir la qualité de représentant de l'employeur ${ }^{67}$. Vis-à-vis la hiérarchie, il représente l'assemblée départementale ${ }^{68}$. La situation est identique pour les vice-doyens. Ces derniers n'assument qu'une fonction technique et professionnelle : ils coordonnent les activités relatives aux programmes d'études. Ces programmes d'études sont fixés par des modules composés de professeurs et d'étudiants. Le vice-doyen administre une famille de modules dans un contexte d'absence de pouvoir décisionnel réel ${ }^{69}$. Il demeure un «chargé d'exécution», un salarié 70 .

La notion de «collégialité » fait donc ressortir des distinctions fondamentales entre la conception de l'aménagement des structures où elle prend place et l'aménagement traditionnel. Dans l'organisation hiérar-

65 Syndicat des professeurs de l'Université du Québec (Montréal) et Université du Québec à Montréal et Association des professeurs de l'Université du Québec et Université du Québec (siège social), (1971) T.T. 216 (confirmant 1971 C.E. 18).

66 Ibidem.

67 Syndicat canadien des employés du transport routier, loc. 455, et Québec Steel Products Ltd. (Produits d'acier du Québec Limitée), (1971) C.E. 523, à p. 524 - Phillips Security Agency Inc. et Union des employés de service, (1972) T.T. 374 - Service social de l'Outaouais Inc. et le Syndicat des professeurs du Service Social de l'Outaouais, (1972) T.T. 63.

68 Voir note 65.

69..Ibidem.

70 Ibidem. 
chique de type traditionnel le supérieur détermine les objectifs, surveille, contrôle, sanctionne et récompense. Dans l'organisation collective, le supérieur devient un animateur: il participe au groupe, planifie, coordonne et sert d'intermédiaire au groupe face à l'employeur qui lui-même est, à la limite, intégré au «groupe social ». En un mot, ces nouveaux professionnels de l'animation, de la coordination, de la planification et de la consultation ne peuvent plus être rattachés à la gérance parce qu'ils ne sont plus les élus de l'employeur mais personnifient et représentent le « groupe social».

En définitive, lorsque le pouvoir décisionnel est exercé en conformité d'une délégation d'autorité patronale les tribunaux acceptent de procéder à la qualification d'un employé comme membre de la gérance au sens de l'article $1 \mathrm{~m}) 1^{\circ}$ du Code du travail. Encore faut-il que la délégation soit réelle et non pas seulement apparente. C'est là poser le problème de l'exercice avec la liberté de manoeuvre caractéristique de l'employeur.

\section{IV - LES CONDITIONS D'EXERCICE DE L'AUTORITÉ}

Parce que la délégation de l'autorité patronale a pour cbjectif ultime de simplifier les liaisons entre l'employeur et les exécutants, la présence d'un ou plusieurs membres de la gérance sera conditionnée par différents facteurs comme la nature de l'entreprise, son envergure, etc. Mais il n'y aura de cadre hiérarchique que dans la mesure où le présumé collaborateur de l'employeur peut agir avec une liberté de manoeuvre formulée en termes de mesure appréciable d'initiative, de responsabilité et de jugement (en A). De plus, parce que la transmission du commandement postule un contrôle qui permet aux titulaires de l'autorité hiérarchique aux niveaux les plus élevés de la hiérarchie de s'assurer successivement de la réalisation de leurs directives aux niveaux inférieurs, l'évaluation de l'existence de la liberté de manoeuvre doit tenir compte de la technique administrative de contrôle appelé contrôle résiduel (en B).

\section{A) Le principe de la nécessité de l'exercice de l'autorité avec une " mesure appréciable d'initiative, de jugement et de responsabilité "}

Toute prise de décision qui, à quelque niveau que ce soit dans l'entreprise, contribue à la réalisation de ses objectifs fait d'un employé un représentant de l'employeur, un membre de la gérance, si cette prise de décision donne lieu à une «mesure appréciable de responsabilité, d'ini- 
tiative et de jugement ${ }^{71}$. Initiative, c'est-à-dire pouvoir d'entreprendre et d'agir soi-même. Jugement, c'est-à-dire possibilité de prendre la décision en dehors de normes rigides pré-établies, programmées et d'un contrôle étroit de l'autorité supérieure. Responsabilité, c'est-à-dire devoir de répondre personnellement à ses supérieurs des décisions prises et qui obligent les subordonnés. Dans la mesure où l'employé ne bénéficie pas de cette marge d'initiative, de responsabilité et de jugement, il doit être considéré comme un salarié ${ }^{72}$. Il faut donc que l'employé agisse avec liberté de manoeuvre en regard soit de la formulation, de la mise en application ou encore de la surveillance des décisions qui engagent l'entreprise. Il faut qu'il règle lui-même les problèmes ${ }^{73}$, qu'il échappe au supérieur immédiat ${ }^{74}$. Il s'impose donc que l'employé règle lui-même les problèmes ou en collaboration étroite avec ceux qui ont la responsabilité d'administrer ${ }^{75}$.

L'exercice de l'autorité hiérarchique avec liberté de manoeuvre peut s'étendre du gérant à un quelconque représentant de l'employeur dans ses relations avec ses salariés. En effet, si on fait appel à l'entreprise comme un «système», les éléments organisationnels de l'entreprise peuvent être considérés comme des «sous-systèmes » du système global. Les soussystèmes peuvent être organisés de façon identique, en l'occurence en unités de travail. À la limite, ces unités, représentées par des équipes ou

71 Hôtel-Dieu d'Arthabaska et le Syndicat des employés d'hôpitaux et d'hospices du comté d'Arthabaska, (1970) T.T. 31 - Institut Doreo Inc. et Syndicat national des employés de l'Institut Doreo Inc., (1972) C.E. 141. Voir de même la jurisprudence citée aux notes 32 à 34 .

72 Ibidem. - Syndicat national des employés de la Crèche d'Youville et la Crèche d'Youville, (1971) T.T. 50, confirmant (1970) C.E. 370.

73 Hôpital du Saint-Sacrement et Alliance professionnelle des para-médicaux de Québec, (1974) T.T. 201, à p. 205.

74 Cité de Charlesbourg et Syndicat des employés municipaux de la Cité de Charlesbourg, (1974) T.T. 414, à p. 415.

75 Ibidem. On notera cependant une décision récente qui va à l'encontre dı courant jurisprudentiel : La Compagnie d'assurance C.N.A. et Union Internationale des employés professionnells et de bureau, loc. 57, (1974) T.T. 220. Dans cette affaire, une accréditation fut accordée pour des employés ayant fait l'objet d'une description de fonctions comportant les tâches suivantes: «C'est au personnel technique et spécialisé qu'incombe la responsabilité de formuler les décisions qui engagent la compagnie dans chacun des cas qui lui sont confiés : il doit alors faire preuve d'une indépendance de jugement et prendre des responsabilités parfois lourdes de conséquences, (...) (à p. 222). Voir le commentaire fait à la note 83 sur cette approche. 
groupes de travail, peuvent être dirigées par de véritables titulaires de l'autorité patronale. La solution nécessite une démarche à deux temps. Il faut d'abord rechercher quel est le but ou l'objectif de l'unité en cause, quel est son rôle, quelle compétence la particularise. S'agit-il de simples travaux d'exécution, de vérification, de mise en application, de travaux de routine ou de travaux créateurs et composant à la finalité de l'entreprise ? C'est en regard de ces travaux que l'on pourra en un premier temps admettre ou non la possibilité de conclure que le responsable de l'unité participe à la gérance ${ }^{76}$. Il conviendra ensuite de rechercher si les responsables des unités ont la mesure appréciable d'initiative, de jugement et de responsabilité qui est exigée. C'est à ce niveau que les chefs de groupe, assimilés par la science administrative à des dirigeants, pourront n'être aux yeux de la science juridique que de purs exécutants, tout comme ils pourront être des membres de la gérance. En effet, l'évaluation de la liberté de manoeuvre à un quelconque niveau d'action s'effectue par l'analyse du contrôle résiduel de l'autorité hiérarchique supérieure sur le niveau présumément hiérarchique suivant.

\section{B) L'impact du contrôle résiduel sur l'évaluation de la liberté de manoeuvre}

Parce qu'un membre de la gérance à un palier d'exercice est toujours responsable envers les paliers supérieurs et a autorité sur les paliers inférieurs, il doit s'assurer de la réalisation des objectifs dont il assume la finalisation. À cet effet, il dispose d'un contrôle résiduel sur les actes de ses subalternes. Ce contrôle consiste dans le pouvoir de mesurer ou comparer les résultats obtenus dans la poursuite des objectifs fixés et de corriger les déviations. Il permet à la gérance de s'assurer que les objectifs seront atteints tel que prévus et que les activités déviées de leur fin seront redressées afin que les performances répondent aux normes souhaitées. Le contrôle n'a en définitive pour objet que l'évaluation et la rectification du rendement des subordonnés dans le processus de l'obtention des fins poursuivies. À l'intérieur de la conduite des opérations, au plan des techniques, ressources et moyens d'action, le délégué doit pouvoir agir avec une marge appréciable d'autonomie, de liberté et de discrétion. Le contrôle résiduel laisse voir que l'existence des véritables cadres hiérarchiques cesse au niveau d'exercice qui est vidé du contenu substantiel et caractéristique de la gérance, à savoir la liberté de manoeuvre. L'appréciation de cette marge d'autonomie a donné lieu à une distinction importante entre les décisions dites «programmées» et les

76 Association nationale des employés de l'alimentation du détail de Québec Inc., et J. B. Cloutier Inc., (1969) D.C.R.T. 9. 
décisions dites «non-programmées» que doit mettre en application un subalterne ${ }^{76 a}$.

\section{LES DÉCISIONS PROGRAMMÉES}

Les décisions programmées ont été perçues par les tribunaux comme étant toutes ces décisions qualifiables de décisions récurrentes et de routine, décisions qui peuvent être traitées selon une procédure définie ou en application de normes fixes et pré-établies. Ceux qui ont à prendre de semblables décisions ne sont pas considérés comme titulaires de l'autorité hiérarchique. À titre d'exemple de décisions programmées, signalons les situations suivantes accomplies par un soi-disant représentant de l'employeur : le fait de faire passer des examens aux candidats à un emploi, d'assurer ultérieurement l'entraînement du personnel et de voir à la distribution et la surveillance du travail dans un contexte qui ne fait appel à aucune initiative personnelle ou à une possibilité d'interprétation en regard des politiques établies 77 ; le fait de vérifier la solvabilité des clients aux fins de consentir des prêts monétaires ou des ventes à crédit sur la base du rapport de crédit selon des critères pré-établis ${ }^{78}$; le fait de compiler des statistiques, de choisir ce qui doit faire ou ne pas faire l'objet de la compilation, et de donner les résultats de la compilation sans pouvoir les interpréter 79 ; le fait de prendre des décisions relativement à l'admission de visiteurs ${ }^{80}$; le fait de disposer des problèmes routiniers de

76a Le Soleil Ltée et le Syndicat des journalistes de Québec inc. (1973) T.T. 292.

77 Syndicat national des employés de commis de bureau du Comté Lapointe c. Évaluation technique Inc., (1970) C.E. 566 - Union des employés de commerce, loc. 500 R.C.I.A. c. Renommée Inc., (1970) C.E. 317.

78 Union des employés de commerce c Renommée Inc., (1970) C.E. 317 - Syndicat national des chefs d'équipes des magasins Lamontagne Ltée c. Lamontagne Ltée. (1970) C.E. 530.

79 American Biltrite Rubber Co. (Canada) Ltd. et Ouvriers unis du caoutchouc, de Sherbrooke Inc., (1973) T.T. 244 à p. 248. Cité de Charlesbourg et Syndicat des employés municipaux de la Cité de Charlesbourg, (1974) T.T. 414, à p. 415. Syndicat national des employé de Lake Asbestos of Quebec et Lake Asbestos of Quebec, (1970) T.T. 39. - Union locale 919 des ouvriers unis du caoutchouc, liège, linoléum et plastique d'Amérique et The Goodyear Tire Co. Ltd., (1971) C.E. 911.

80 Syndicat national des employés de Lake Asbestos of Quebec c. Lake Asbestos of Quebec Ltd., (1970) T.T. 1 - Régie de la Place des Arts et Construction and Supply Driver Allied Workers, loc. 903, et Suzan Lavender, (1971) T.T. 249 Syndicat des agents de sécurité de C.I.L. de Nitro v. C.I.L. Explosive de Salaberry Works Nitro, (1971) T.T. 134 - Union des employés de service d'édifices, loc. 298, c. Édifice de la Prévoyence Inc., (1969) D.C.R.T. 346. 
son supérieur en son absence ${ }^{81}$; la simple distribution technique du travail ${ }^{82}$, etc.

\section{LES DÉCISIONS NON-PROGRAMMEES}

Les décisions non-programmées sont toutes ces décisions par lesquelles un individu peut apporter un apport personnel dans la poursuite des objectifs. Pour que cet apport puisse permettre à l'employé de se réclamer du statut de membre de la gérance, il est nécessaire qu'il se concrétise en une prise de décision comportant trois caractéristiques essentielles : participation personnelle de l'employé à la formulation de la réalisation de l'objectif particulier qu'il doit assumer; un libre choix des voies et moyens d'action pour atteindre cet objectif ; le pouvoir de décider s'il agit dans l'intérêt de l'entreprise ${ }^{83}$. Dans la mesure où les décisions prises par un présumé collaborateur de l'entreprise ne deviennent exécutoires que si le supérieur immédiat les sanctionne, le présumé collaborateur n'est qu'un exécutant ${ }^{84}$. Ainsi, à titre d'exemple, pour être

81 Commission des écoles catholiques de Shawinigan c. le Syndicat national des employés de bureau de la Commission scolaire de la Mauricie (C.S.N.) (1970) C.E. 173 - Syndicat des employés de la Commission Scolaire Régionale Lanaudière et Commission Scolaire Lanaudière, (1972) C.E. 104.

82 Dufour Ready Mix Inc. et Syndicat des employés de Dufour Ready Mix Inc., (1974) T.T. 65 à p. 66.

83 On notera que la décision récente signalée à la référence 75 (Cité de Charlesbourg) est peut-être celle qui a le mieux rendu cette nécessité d'une «mesure appréciable d'initiative, de jugement et de responsabilité ». L'approche jurisprudentielle courante est cependant plus conservatrice : à titre d'illustration on pourra consulter : Alliance des infirmières de Montréal et Hôpital Charles Lemoyne, (1970) C.E. 499 - Ville de Hauterive et Syndicat national des employés de bureau de la Ville de Hauterive, (1971) C.E. 765 - Union des employés de commerce et Marché Victoria, (1970) C.E. 434 - Hôpital St-Eusèbe de Joliette et Syndicat catholique nationale des employés des institutions religieuses du diocèse de Joliette, (1970) T.T. 413 - Union internationale des employés professionnels et de bureau et Commission Scolaire régionale de Chambly, (1971) C.E. 97. Dans ces dernières affaires, on a conclu à la qualité de membres de la gérance pour des employés dont les pouvoirs n'étaient pas plus importants que ceux constatés dans le cas Charlesbourg.

84 Syndicat des employés des commis de bureau du Comté Lapointe c. Évaluation technique, (1970) C.E. 566 - Union des employés de commerce, loc. 500, R.SC. I.A. c. Renommée Inc. (1970) C.E. 317 - La Commission des écoles catholiques de Shawinigan c. Syndicat national des employés de bureau de la Commission scolaire de la Mauricie, (1970) C.E. 173, à p. 176 - Régie de la Place des Arts et Construction \& Supply Drivers \& Allied Workers, loc. 903, (1971) T.T. 249. 
identifié à la gérance, l'employé qui dirige un groupe de salarié doit être autorisé à donner des ordres, modifier les conditions de travail, imposer des sanctions disciplinaires et faire des réprimandes ou recommandations en cette matière qui auront force exécutoire ${ }^{85}$. Il est nécessaire que les recommandations soient le critère direct, immédiat et déterminant concernant des promotions, rétrogradations, embauchages et mesures disciplinaires ; le simple fait de rapporter des agissements qui éventuellement permettront de conduire à des mesures disciplinaires n'est pas suffisant pour prétendre représenter l'employeur. En d'autres termes, il ne suffit pas de poser des gestes qui peuvent conduire à des mesures disciplinaires vis-à-vis d'autres employés ${ }^{87}$. Donc si des employés possèdent une certaine latitude dans l'exécution de fonctions qui présument l'exercice du pouvoir de commandement mais qu'un contrôle constant s'exerce de la part du supérieur immédiat, ces employés restent des salariés ne participant pas à la gérance ${ }^{88}$.

En conclusion sur la condition d'appartenance au groupe personnifiant l'employeur, on doit rappeler que ce n'est que dans la mesure où un employé exerce le «pouvoir de commandement» ou «autorité hiérarchique » avec une mesure appréciable d'iintiative, de jugement et de responsabilité que cet individu est visé par l'article $1 \mathrm{~m}) 1^{\circ}$ du Code $d u$ travail. Cet exercice pourra s'effectuer dans un quelconque mode de structure qui encadre l'autorité patronale. On parlera alors des niveaux d'exercice, matière qu'il convient maintenant d'aborder.

\section{$V$ - LES PALIERS D'EXERCICE DE L'AUTORITÉ PATRONALE}

La notion de membres de la gérance, qui est à peu de chose près synonyme de celle de cadres hiérarchiques, a été conçue pour identifier ceux qui ont, par délégation, vocation à détenir l'autorité hiérarchique dans l'entreprise. Elle exclut de sa compréhension celle de cadres-con-

85 Syndicat national des employés de la Crèche d'Youville et la Crèche d'Youville, (1971) T.T. 50.

86 Construction and Supply Drivers \& Allied Workers, loc. 903, la Régie de la Place des Arts, Suzan Lavender, (1971) T.T. 249 - Syndicat national des employés de l'Institut Philippe Pinel c. Institut Philippe Pinel, (1972) T.T. 67.

87 Syndicat national des employés de Lake Asbestos of Quebec c. Lake Asbestis of Quebec Ltd., (1970) T.T. 1.

88 Union des employés de commerce, loc. 500, R.C.I.A. c. Marché Victoria Inc., (1970) C.E. 434 - Le Soleil c. Le Syndicat des journalistes de Québec Inc., (1970) C.E. 306, aux pp. 307 et 308 - Syndicat national des employés de Fournier Steel Works Ltd. c. Fournier Steel Works Ltd., (1970) C.E. 503. 
seils. C'est une notion qui, en regard des opérations et des activités quotidiennes d'une entreprise, concrétise le patronat nominal ou patronat de fonction. Ce patronat peut avoir plus ou moins d'extension selon le niveau d'action qui concrétise la répartition de l'autorité patronale. Les auteurs ont tenté de mouler la réalité de leurs attributions et de leurs niveaux d'action en distinguant trois types de cadres hiérarchiques: les cadres supérieurs ou gérants (higher level management), (en A); les cadres intermédiaires ou surintendants (middle management) (en B); et les cadres subalternes ou contremaîtres et autres représentants de l'employeur dans ses relations avec ses salariés (first line management) (en C)

\section{A) Les cadres hiérarchiques supérieurs (gérants)}

La qualification de gérant est réservée à l'employé qui administre pour un employeur ou sous sa direction un commerce, une entreprise, etc. ${ }^{90}$. Elle vise de même celui qui gère un département, un service, etc., et qui relève de l'autorité d'un gérant-général ${ }^{91}$. La notion a à peu près le même sens et la même portée que l'expression cadre hiérarchique. Cette dernière désigne l'employé qui seconde directement les plus hautes instances ou organes de l'entreprise. Les instances secondées peuvent être un conseil d'administration, un conseil des ministres, une commission de gestion, un administrateur, un ministre, etc. Le cadre supérieur est responsable devant ces organes. C'est donc un collaborateur des administrateurs pouvant participer à l'élaboration des directives et qui est chargé de les faire appliquer et d'en contrôler la réalisation. On catalogue ordinairement parmi les cadres supérieurs les différents directeurs généraux spécifiques, les hauts fonctionnaires, notamment les sous-ministres, etc. ${ }^{92}$. Les cadres supérieurs sont quelquefois qualifiés cadres-dirigeants (ou cadres de direction). Sous cet angle, le terme cadre hiérarchique supérieur est synonyme de l'expression gérant-général. Les attributions de ce dernier n'ont de limites que celles de l'employeur lui-même devant qui il est responsable pour les activités de l'entreprise. La notion cadre supérieur s'entend aussi de l'employé qui n'est pas responsable devant les instances supérieures mais qui par contre seconde les cadres-dirigeants qui le sont ${ }^{93}$

89 Voir les auteurs cités à la note 22.

90 Laiterie Perrette Ltée et J. Campeau, (1973) T.T. 141, à p. 148.

91 Union des employés de commerce, loc. 500, et Provigo Montréal Inc., (1974) T.T. 382, à p. 384.

92 Voir les auteurs cités à la note 22 .

93 IIbidem. 
Les fonctions assumées par les cadres supérieurs ou gérants sont de nature diverse mais seul l'exercice du pouvoir décisionnel avec initiative, jugement et responsabilité les spécifie en regard de l'interprétation de l'article $1 \mathrm{~m}$ ) $1^{\circ}$ du Code. Ces cadres se caractérisent par le pouvoir de commandement sur de grands ensembles ou en fonction de l'élaboration de politiques générales à long terme. Dans l'exercice du pouvoir de commandement, les cadres supérieurs peuvent n'être en relation qu'avec les collaborateurs extérieurs de l'entreprise, comme par exemple les sous-contractants, les fournisseurs de capitaux, etc. Ils peuvent de même ne faire affaire qu'avec les clients ou le public. Généralement ils seront en relation directe ou indirecte avec le personnel même, salarié ou non, de l'entreprise. En un mot, pour reprendre les propos de J.P. Runkle :
«In fact, a manager may be a manager without any employees under him at all; for he may direct and control a department or an operation or phase of the business by dealing directly with person other than employees of his employer $\gg 94$.

Cette perception est conforme à une abondante jurisprudence ${ }^{95}$.

\section{B) Les cadres hiérarchiques intermédiaires (surintendants)}

Les cadres supérieurs sont normalement assistés d'employés qui sont appelés à prendre, dans des secteurs d'activités spécifiques, des décisions à moyen terme qui, dans le temps, normaliseront les opérations et activités de l'entreprise. L'autorité de ces employés, qualifiés de cadres intermédiaires, s'exerce sur un personnel réduit. On qualifie ordinairement de cadres intermédiaires les directeurs ou chefs de service ou de division, les surintendants, etc. ${ }^{96}$. La présence de cadres intermédiaires tient du fait qu'une structure peut être plus ou moins aplatie. Le principe du contrôle résiduel que détient un supérieur hiérarchique a comme corrolaire qu'il existe une limite au nombre d'exécutants ou de collaborateurs qu'un

94 The Buyer as a Depend Manager, op cit., note 52, à p. 36.

95 J. B. Laliberté Limitée et Association des Commis de détail, (1967) R.D.T. 246 - The Montreal Newspaper Guild, loc. 111, et Montreal Star Co. Ltd., (1964) R.D.T. 532 - R.C.A. Limited et R.C.A. Engineers and Scientists Association, (1971) T.T. 14 - Le Syndicat canadien de la fonction publique et Ville de Vimont, (1966) R.D.T. 282 - Association des principaux d'écoles de Québec et Commission des écoles catholiques du Québec, (1966) R.D.T. 321 - Hôtel-Dieu d'Arthabaska, (1970) C.E. 181 - Syndicat catholique des employés municipaux de Sillery et Cité de Sillery, (1970) C.E. 407.

96 Voir les auteurs cités à la note 22 . 
supérieur peut superviser. Partant, dans la mesure où l'entreprise est le lieu d'action d'un personnel nombreux, il sera nécessaire de répartir l'autorité patronale entre des titulaires pouvant soit s'adresser directement aux exécutants, soit s'adresser à des intermédiaires qui eux-mêmes pourront soit s'adresser aux exécutants, soit à d'autres intermédiaires.

L'autorité des cadres intermédiaires porte d'ordinaire en regard de décisions à moyen terme qui, dans le temps, normaliseront les opérations et activités de l'entreprise. Les pouvoirs qu'ils peuvent exercer en termes de techniques administratives varient et sont fonction de la nature et de la dimension de l'entreprise ${ }^{97}$. Ils pourront posséder un ensemble de pouvoirs tenant à l'organisation de la production, à l'embauchage et à l'entraînement du personnel, à l'utilisation de la main-d'oeuvre et à l'évaluation de celle-ci, à la direction, surveillance et contrôle des activités, aux mesures administratives et disciplinaires et autres attributs ${ }^{98}$. Pour les fins de l'article $1 \mathrm{~m}$ ) $1^{\circ}$, ces attributs ne sont pris en considération que s'ils sont sous-tendus par l'exercice du pouvoir décisionnel avec liberté de manoeuvre ${ }^{99}$.

\section{C) Les cadres subalternes (contremaîtres ou représentants de l'employeur dans ses relations avec ses salariés)}

Les cadres intermédiaires sont généralement secondés dans l'exercice de leurs fonctions par des agents de maîtrise ou cadres subalternes. Ces derniers sont caractérisés par la prise de décision rapide au niveau de l'action quotidienne ou à court terme, dans des sphères d'activité et

\section{Ibidem.}

98 Institut Mont d'Youville et Syndicat des employés du Mont d'Youville, (1973) T.T. 471. Syndicat professionnel des diététistes du Québec et Hôpital HôtelDieu de Montréal, (1972) C.E. 299 - Syndicat des employés de bureau des écoles catholiques de Chicoutimi c. La Commission des écoles catholiques de Chicoutimi, (1971) C.E. 230 - Syndicat national des employés de commerce et de bureau du comté Lapointe c. Évaluation technique Inc (1970) C.E. 566 - Syndicat des ouvriers de la Régie des Alcools du Québec (C.S.N.) c. La Régie des Alcools du Québec( (1970) C.E. 365 - Syndicat des fonctionnaires municipaux de la Cité de Ste-Foy c. Cité de Ste-Foy, (1971) C.E. 897 - Ville de Ste-Foy c. Syndicat des fonctionnaires de la Ville de Ste-Foy, (1967) R.D.T. 205 - J.B. Laliberté c. Association des commis de détail, (1967) R.D.T. 246 - Métallurgistes Unis d'Amérique c. Continental Can. Co. of Canada Ltd., (1969) R.D.T. 221 Fraternité canadienne des cheminots, employés de transport et autres ouvriers c. Beton Dynamique Limitée, (1970) C.E. 336 - P. Fisher c. La Centrale du Livre Inc., (1969) D.C.R.T. 213.

\section{Ibidem.}


sur des équipes de travail réduites. Sont en général qualifiés de cadres subalternes en science administrative les contremaîtres, chefs d'équipe, commis-chef de bureau ${ }^{100}$. Ce ne sont cependant pas tous les cadres subalternes au sens de la science administrative qui peuvent être qualifiés de cadres hiérarchiques au sens du Code. Les tribunaux ont en effet donné un sens précis à l'expression « représentant de l'employeur dans ses relations avec ses salariés ». Ce sens ne permet pas de comprendre l'expression comme terme générique ou collectif des chefs d'équipe, commis de bureau et autres cadres subalternes de la science administrative. Il nous apparaît important de préciser les cadres subalternes visés par l'article $1 \mathrm{~m}$ ) $1^{\circ}$ du Code du travail, puis de cerner le sens et la portée de l'expression ou représentant de l'employeur dans ses relations avec ses salariés.

\section{LE DERNIER NIVEAU D'EXERCICE DE L'AUTORITÉ PATRONALE}

Tout cadre hiérarchique de la gérance subalterne, et quel que soit son titre, est visé par le texte $1 \mathrm{~m}$ ) $1^{\circ}$ s'il exerce, par délégation, l'autorité patronale avec jugement, initiative et responsabilité. Cette affirmation trouve son fondement et sa justification dans le critère de fond que les tribunaux utilisent pour procéder à la qualification des employés dits collaborateurs de l'employeur ou employés identifiés aux intérêts de la partie patronale dans l'aménagement des rapports collectifs du travail. Pourtant, en dépit du principe qui veut que l'on ne s'occupe pas de titres mais uniquement des fonctions et responsabilités réellement exercées, les commissaires-enquêteurs et le tribunal du travail ont à maintes reprises dégagé une déclaration d'accommodement aux termes de laquelle les chefs d'équipe ne sont pas assimilables aux cadres hiérarchiques visés par le Code ${ }^{101}$. Les juridictions antérieures, notamment la Commission

100 Voir les auteurs cités à la note 22 .

101 Commission des écoles catholiques de Montréal et Association professionnelle du personnel administratif de la Commission des écoles catholiques de Montréal, (1971) T.T. 239 - Pasolds (Canada) Ltd. et Ouvriers unis des textiles d'Amérique, (1970) T.T. 28 - Syndicat national des employés de commerce et de bureau du comté Lapointe Inc. et F.J. Lussier Inc., (1970) C.E. 599, à p. 600 - Fraternité internationale des travailleurs en électricité et Montreal Answering Service, (1971) C.E. 69 - Syndicat des employés de Montreal Magog Inc. et Montreal Newspaper Printing Pressmen's Union, loc. 41, et Imprimerie Montreal Magog, (1972) C.E. 131 - Sir George William University et National Union of Employer of Sir George William University, (1971) C.E. 684. 
des relations du travail, avaient pour leur part conclu en sens inverse ${ }^{102}$. L'une et l'autre de ces déclarations reflètent mal, selon nous, la réalité des faits que ces tribunaux ont été appelés à étudier. Dans le contexte particulier où ces déclarations apparamment contradictoires ont été formulées, elles restent fondées; elles ne le sont pas cependant en tant que principe d'interprétation qui se veut représentatif de l'économie du Code. Ainsi, par exemple, les déclarations des commissaires-enquêteurs et du tribunal du travail ont toujours été faites en regard de chefs d'équipe à autorité professionnelle ou technique ${ }^{103}$, tandis que celles de la Commission des relations du travail l'ont été en regard d'espèces jurisprudentielles mettant en cause des chefs d'équipe titulaires de l'autorité hiérarchique ${ }^{104}$. Nous soutenons pour notre part que sont visés tous les cadres qui, indépendamment de leurs titres et de leurs niveaux d'action, personnifient réellement l'employeur.

En effet, s'il est exact que l'employeur ne peut pas «éparpiller l'autorité sans égard à la raison et sans droit de regard sur personne » ${ }^{105}$, il est aussi exact que les autorités judiciaires ne peuvent pas intervenir en regard d'une délégation réelle et permise de l'autorité patronale ${ }^{106}$. Ils ne

102 Association des Éducateurs de Pont-Viau, dossier de la C.R.T. no. A-65725, décision du 13 mars 1965, non publiée (sommaire in Descôteaux G., Répertoire des décisions en application du Code du travail, 1970, Université d'Ottawa, à p. 107) - Syndicat professionnel des infirmières catholiques, dossier de la C.R.T. no. A-66-377, décision du 26 août 1966, non publiée, (sommaire in Descôteaux, op. cit., à p. 108) - Syndicat des employés des hôpitaux de Montréal Inc., dossier de la C.R.T. A-66-419, décision en date du 6 octobre 1966, non publiée, (sommaire in Descôteaux, à p. 111) - Hôtel-Dieu Notre-Dame de Beauce, dossier de la C.R.T. no. A-66-627, décision du 25 mars 1966, non publiée (sommaire in Descôteaux, à p. 113) - Hôpital St-Joseph, Beauceville, dossier de la C.R.T. A-68-1404, décision non publiée, 5 avril 1968 (sommaire in Descôteaux, à p. 121) - J. B. Laliberté et Association des commis de détail, (1967) R.D.T. 246 - Association des principaux d'écoles de Québec et la Commission des écoles catholiques de Québec, (1966) R.D.T. 321. Contra : Métallurgistes unis d'Amérique, loc. 4278, Rouyn et Noranda Mines Ltd., (1969) D.C.R.T. 232.

103 Voir la jurisprudence citée à la note 101. Voir de même Service familial de la Rive-Sud et Syndicat des employés du Service familial de la Rive-Sud, et Syndicat des professionnels du Service familial de la Rive-Sud, (1972) T.T. 71 Salaison Her-Pack et les Travailleurs canadiens de l'alimentation et d'autres industrie, (1971) T.T. 151.

104 Voir le jurisprudence citée à la note 102 .

105 Syndicat professionnel des diététistes de Québec et Hôpital Hôtel-Dieu de Montréal, (1972) C.E. 299, à p. 307.

106 Hôpital Saint-Rédempteur de Matane et le Syndicat des employés de l'hôpital de Saint-Rédempteur de Matane, (1972) C.E. 73. Ville de Montréal et Syndicat des fonctionnaires municipaux de la Ville de Montréal, (1974) T.T. 410. 
peuvent que vérifier la nature des fonctions et responsabilités effectivement accomplies. La présence de véritables cadres hiérarchiques à un niveau inférieur à celui de contremaître tient au fait que la répartition réelle de l'autorité patronale à travers une structure peut-être plus ou moins aplatie en raison de la taille de l'entreprise ou de ses structures. $\mathrm{Si}$ l'on préfère, il est possible que des chefs d'équipe soient titulaires de la même autorité que celle des contremaîtres. Nous croyons que cette approche explique pourquoi certains auteurs ont affirmé que le groupe de la direction inclut tout employé qui exerce l'autorité patronale à quelque niveau que ce soit ${ }^{107}$. Mais il faut que cet employé exerce avec liberté de manoeuvre l'autorité patronale si bien que l'on ne peut pas être d'accord avec l'affirmation qui veut que l'exercice de la moindre parcelle d'autorité ${ }^{108}$ permet de déceler un cadre hiérarchique. En somme, les cadres subalternes doivent s'entendre non seulement des contremaîtres mais aussi des responsables et autres employés qui exercent véritablement l'autorité patronale à un niveau quelconque de la hiérarchie dans l'entreprise ${ }^{109}$. Cet énoncé trouve sa véritable raison d'être dans la finale explicative qui apparaît au texte législatif, à savoir «.. ou représentant de l'employeur dans ses relations avec ses salariés ».

L'expression qui termine le texte $1 \mathrm{~m}$ ) $1^{\circ}$ pose de délicats problèmes d'interprétation. On doit d'abord signaler que cette expression n'est pas synonyme notamment de celle de contremaître car le législateur ne parle pas en principe pour ne rien dire ${ }^{110}$. Quel sens et portée doit-on alors donner à cette expression? Doit-on la conceptualiser en fonction de la seule représentation effective auprès des salariés, c'est-à-dire prétendre qu'elle ne vise que les employés impliqués dans la gestion du personnel ou des ressources humaines. Certaines espèces jurisprudentielles le laissent penser en affirmant que le législateur a voulu viser uniquernent les

107 Voir les auteurs cités à la note 22.

108 Voir l'auteur cité à la note 28.

109 Mérillon Canada Ltée et Ouvriers unis du caoutchouc, linoléum et plastique d'Amérique, (1972) T.T. 376 - Bey-Well Food Market Inc., et M. Godin, (1971) T.T. 302 - Syndicat du transport routier Saguenay Lac Saint-Jean et Nil Girard Transport Ltée, (1972) T.T. 249 - Union des employés de commerce, Union des employés de commerce, loc. 500, R.C.I.A. et Renommée Inc., (1970) C.E. 317 - Union des bûcherons et employés de scieries de la Fraternité unis des charpentiers-menuisiers d'Amérique, loc. 2817 et Syndicat forestier de St-Alexis des Monts, (1970) C.E. 649.

110 Union des employés de commerce, loc. 500, et Proviga Montréal Inc., (1974) T.T. 382, à p. 384. 
seuls cas de représentation auprès des salariés et non pas, par exemple, la représentation auprès de la clientèle ou du public ${ }^{111}$. Cette approche ne peut être retenue. Il faut en effet se rappeler que le texte en son entier prend figure d'une définition par énumération. Dans ce style de rédaction législative, le terme ou utilisé entre le dernier et l'avant-dernier terme doit être interprété comme une finale explicative du texte ${ }^{112}$. Si l'on devait accepter que la finale explicative ne permet de comprendre que la représentation effective auprès des salariés, l'on devrait logiquement accepter que l'ensemble du texte vise la seule gestion du personnel ou des ressources humaines puisque la définition par énumération implique qu'en principe on ne doive comprendre que les choses du même genre comme étant visées par le texte ${ }^{113}$. Or le principe de fond dégagé par les tribunaux et l'économie qui sous-tend ce principe ne permettent pas de conclure que l'expression ne vise que la seule représentation effective auprès des salariés.

Rappelons d'abord que l'article $1 \mathrm{~m}$ ) $1^{\circ}$ vise globalement la Gestion, qu'elle s'exprime en termes de gestion financière, gestion de ressources humaines, ou autre. Il vise de même les différentes fonctions patronales (coordination, planification, etc.) sous-tendues par l'exercice de l'autorité patronale. Par la finale explicative, le législateur a indiqué qu'il fallait donner aux différents termes du texte toute l'extension possible. C'est pourquoi certaines espèces jurisprudentielles ont-elles permis de dégager que l'expression comprend toutes les personnes qui, sans avoir la direction effective de salariés, représenteraient l'employeur auprès des salariés : directeur du personnel, de la sécurité, fonds de pension, etc. ${ }^{114}$. Selon nous, le législateur a voulu marquer son souci de n'oublier personne, en l'occurence de n'oublier aucun des employés qui sans avoir la direction de salariés peuvent potentiellement avoir une semblable direction. La finale de l'article $1 \mathrm{~m}$ ) $1^{\circ}$ en est donc une qui tout à la fois explique et complète le sens et la portée de ce texte; elle se comprend non pas en

111 Union locale 919 des ouvriers unis du caoutchouc, liège, linoléum et plastique d'Amérique et The Goodyear Tire Co. Ltd., (1971) C.E. 911, à p. 919 - Syndicat professionnel des diététistes du Québec et Hôpital Saint-Jean de Dieu, (1971) C.E. 635, à p. 640, (1971 T.T. 241) - Boulangerie Guérin et Association des employés de la Boulangerie et Pâtisserie de Drummondville, (1972) C.E. 257.

112 L. P. PIGEON, Rédaction et interprétation des lois, op. cit., note 3, aux pp. 22, 28, 38 .

113 Ibidem, à p. 38.

114 Syndicat professionnel des diététistes du Québec et Hôpital Hôtel-Dieu de Montréal, (1972) C.E. 299. à p. 303. Cité de Charlesbourg et Syndicat des employés municipaux de la Cité de Charlesbourg, (1974) T.T. 414, aux pp. 415, 416. 
soi mais en regard et par le critère de fond dégagé par les tribunaux, l'exercice de l'autorité patronale avec liberté de manoeuvre à tout niveau et au dernier niveau.

\section{CONCLUSION}

En conclusion sur la notion de l'employé visé par l'article $1 \mathrm{~m}) 1^{\circ}$ du Code du travail, on doit rappeler que les tribunaux font appel à un critère de fond, tiré de l'examen même des fonctions et responsabilités réellement assumées, pour cerner le sens et la portée du texte. L'article ne s'adresse qu'aux titulaires de l'autorité hiérarchique, laquelle ne peut exister que dans la mesure où l'employé exerce, par délégation, la fonction de commandement (pouvoir décisionnel) avec initiative, jugement et responsabilité. En terminant, nous tenons à justifier le pourquoi de cette analyse qui pourra être qualifiée par certains d'essentiellement descriptive. Nous avons cru opportun de bien caractériser les employés visés par le texte avant de nous interroger sur leur statut juridique au sens du Code du travail, matière qui sera abordée dans une prochaine étude. Plusieurs auront en effet remarqué qu'on assiste depuis quelques années à une prolifération d'écrits sur le syndicalisme des cadres. La plupart de ces études, bien qu'elles remettent directement ou indirectement en cause l'économie du Code, ne font que des références nominatrices à la législation sans préciser qui est réellement visé. Nous nous sommes proposés dans cet essai de mettre l'accent sur ce problème, nous engageant dans une voie qui nous l'espérons apportera une contribution utile aux études sur les cadres tels que compris par le Code du travail.

\section{Line Management under the Quebec Labor Code}

The Labor Code states that the word employee does not include « a person who, in the opinion of the investigation commissionner, is employed as manager, superintendant, foreman or representative of the employer in his relations with his employees». To undertake the identification of employees affected by this definition, labor courts do not care much about job titles, but rather take a criterion as a basis : freedom of action in exercising management authority. Employees carrying on such a form of authority are called management members or line management.

Job Titles mentioned in Section $1 \mathrm{~m}$ ) $1^{\circ}$ of the Labor Code are unimportant in themselves according to labor courts. Jobs fulfilled by an employee and the nature of his responsabilities determine if he belongs to line management. Hence 
two consequences are issued. On the one hand the probatory force of job titles is, in the views of the labor courts, very restricted and sometimes rejected by labor courts. On the other hand the agreement between parties on the nature of a given job does not deprive labor courts to study all affective angles of the work done.

The criterion used in qualifying the lines of authority finds its foundation and its meaning in the historical back-ground. The Labor Code as well as the Labor Relations Act keeps up and uses the organizing principles of enterprises at time of industrial production. Enterprises must have a two level working structure : management and operations. Management includes all persons having management prerogatives. So that a vague form of authority could be qualified as one with managerial prerogatives, three requirements are necessary: it must be of a certain nature ; it must act off the authority of a particular relevant source : and it must be exercised in accordance with specific conditions. Let us analyse those requirements.

Because the historic back-ground does not permit to cover through Section $1 \mathrm{~m}) 1^{\circ}$ the members of scientific management of enterprises labor courts have decided that a manager could exist insofar as an employee commands and is part of management. To command implies decisive action through which top management releases and directs the activities of lower level employees towards a common goal. It is the fact of decision-making that compels and coerces. Authority then justifies such actions. This kind of authority is specific to the employer and qualifies is hierarchical or management prerogatives. Other managerial occupations such as coordination, planning, organization, information and control do not conclude in favour of carrying out formal authority, were it not in cases in which they are supported by a power of command. Necessity to fulfill this commanding function supposes that some forms of authority must be excluded from the complement of the formal authority; we mean professionnal or technical authority of staff management.

An employee wields the employer's authority when he carries on the command on behalf and in the name of his employer. This requirement involves a preliminary authorization to manage. The authorization becomes effective through delegation. Delegation is an act through which an employer transfers part of his prerogatives to his employees. Authority so transferred remains the initial formal authority. It is transferred from higher hierarchical levels to inferior levels. At the legal level, the authority so transferred is monolithic. It must be handed over within the scope of an operational system of delegation and not within the scope of a representative system. In the first instance, the operating chart or the organization lead to think that the employee is authorized to manage as line management; in the second one, the employee manages occasionnally as a supposed line-management. However, the moment an employee acts as line-management when the necessity aroses, he manages within an operational system of delegation. Finally it is suitable to mention that employees backing-up group decision-making way not qualify as managers, as this form of authority leaves-out dualism (managementoperations) recommended by the scientific organization of enterprises at the time of industrial production. 
Delegation of authority does not mean effective carrying out of formal authority. It is essential that the delegate of such handed over authority could manage with an appreciable standard of initiative, judgment and responsibility. Through initiative, the employee will manage and act by himself. Through judgment, he will be capable to render decisions out off rigid and pre-established standard and out off a strict control from formal authority. Through responsibility, he will stand to the management for his decisions which compel his subordinates. Estimation of the margin of autonomy is analysed by labor courts in examining the residual control held by the manager towards his subordinates. This study showed the differences in exercising authority between lines of programmed and unprogrammed decisions. The former do not permit to qualify an employee as manager. If an employee carries out formal authority without restraint, he will be considered as line manager. There are three types of line management: higher level managment, middle management and first line management. Higher level management is clearly market by the command power upon long term general policies. Middle management is involved with middle term activities which standardize the operations of the firm. First line management is noted for its daily or short term decision upon curtailed teamwork.

In short, with a view and scope of Section I m) $1^{\circ}$ of the Labor Code, we wish to underline that this paper affects only the employees entitled to carry on formal authority without restraint upon any hierarchical level of the firm.

\section{LE SYNDICALISME CANADIEN (1968) une réévaluation}

Introduction, Gérard Dion - Les objectifs syndicaux traditionnels et la société nouvelle (Jean-Réal Cardin - Gérard Picard - Louis Laberge - Jean Brunelle. Les structures syndicales et objectifs syndicaux (Stuart Jamieson Philippe Vaillancourt - Roland Martel). La démocratie syndicale (Gérard Dion - Adrien Plourde). Les rivalités syndicales: force ou faiblesse (Evelyne Dumas - Gérard Rancourt - Raymond Parent). Le syndicalisme et les travailleurs non-syndiqués (Léo Roback - Jean-Gérin-Lajoie - F.-X. Légaré). L'extension de la formule syndicale à des secteurs non-traditionnels (Shirley B. Goldenberg - André Thibaudeau - Raymond-G. Laliberté - Jean-Paul Brassard). Le syndicalisme et la participation aux décisions économiques (Bernard Solasse - Jacques Archambeault - Fernand Daoust - Charles Perreault). Les syndicats et l'action politique (Vincent Lemieux - Marcel Pepin - Laurent Châteauneuf et William Dodge). Le syndicalisme, la société nouvelle et la pauvreté (Hon. Maurice Lamontagne). Bilan et horizons. Annexes: Le syndicalisme au Canada; la concurrence syndicale dans le Québec (Gérard Dion).

Prix : $\$ 5.00$

\section{LES PRESSES DE L'UNIVERSITE LAVAL}
Case Postale 2447
Québec 10
Téléphone :656-2131 